\title{
Solving Complex and Disordered Surface Structures with Electron: Diffraction
}

M.A. Van Hove. Materials and Chemical Sciences Division. Lawrence Berkeley Laboratory. University of California. Berkeley. California 94720. USA

The past of surface structure determination with low-onersy electron diffraction (LEED) will be briefiy reviewed, setting the stage for a discussion of recent and future developments. The aim of these developments is to solve complex and disordered surface structures. Some efficient solutions to the theoretical and experimental problems will be presented. Since the theoretical problems dominate. the emphasis will be on theoretical approaches to the calculation of the multiple scattering of electrons through complex and disordered surfaces.

\section{Introduction}

\subsection{The Past}

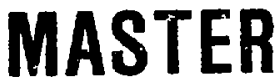

Since its inception as a surface structura! tool in the early 1970's [1-5]. How-energy electron diffraction (LEED) has been used to determine at least $\mathbf{2 0 0}$ structures. These range from simple clean metal surfaces to more complicated metal or semiconductor reconstructions, as wall as molecular adsorbate layera. The vast majority of these structures is of the ordered type, i.e. the structure is based on diffraction spot intensities that correspond to ordered parts of the surface. One structure, that of axyzen on $W\{100\}$, consists of a disordered overlayer and its structuro was obtained vis the diffuse intensity that exists between the diffraction spots [6].

Another 50 or 80 structuses have been determined relatively completely by other techniques of surface structure analysis. Foremost among these are medium- and high-energy ion scattering (MEIS and HEIS). surface extended $x$-ray absorption fine structure (SEXAFS). ancla-resolved photoelectron emission fine structure (ARPEFS) and other variants of pho toelectron diffraction. The structures determined with these techniques include simple and ordered as well as complex and disordered casee. The more complex structures have often been studied incompletely. e.f. with near-adge $x$-ray absorption fine structure (NEXAFS). But there has always been a preference for simple ordered structures with these techniques as well, because of a hisher chance of success and fewer unknown parameters to be determined.

Recently. a database of solved surface structures has been compiled. It comes in the form of a handbook [7] containing a "catalogus" of those structures. as well as in the form of an electronic database with coinputer graphing program [8]. Over 250 structures are included: 
they exhibit the considerable achievements of surface structural analysis over some 15 years. llowever, they also clearly point towards new types of surface structure that were so far considered too complicated to solvo.

The most complex and "complete" structural determinations to date contain 4 to 5 struciural parameters. Most of these structures were analyzed by LEED. Examples are:

- the metal surface reconstructions of $\mathrm{Au}[9]$. Ir [10] and $\mathrm{Pt}\{110\}-(1 \times 2)$ [11], illustrated in Figure 1:

- the structure of $S$ adsorbed on reconstructed $\operatorname{Ir}\{110\}(1 \times 2)$ [12]. shown in Figure 2:

- the reconstruction of $\operatorname{Ir}\{100\}-(1 \times 5)[13]$ :

- the semiconductor reconstructions of $\operatorname{Si}\{100\}-(2 \times 1)[14]$. Si $\{111\}-(2 \times 1)[15]$ and GaAs\{111\}-(2×2) [16]:

- molecular overlayer structures on metal substrates. such as $\operatorname{Rh}[17]$ and $\operatorname{Pd}\{111\}-$ $(3 \times 3)-\mathrm{C}_{8} \mathrm{H}_{6}+2 \mathrm{CO}$ [18], illustrated in Figures 3 and 4, respectively.

This is not to say that other techniques did not contribute to the results. In fact. LEED alone could not have solved many of these structures. Outside input has been essential in eliminating many a prieri possible models. However. One may say that LEED often produces the most accurate final reault for relatively complex structures.

\subsection{The Objective}

Clearly, one would like to solve many more complex structures. For instance: stepped surfaces in the clean state or with adsorbates, large-unit cell semiconductor reconstructions and the effect of adsorbates thereupon. overlayers of larce molecules, disordered overlayers. defect structures in clean or adsorbate-covered surfaces, and incommensurate overlayers.

To be more specific. here is a list of directions in which it is desirable to develop the LEED technique. as well as other surfaca-structure techniques:

- structures with many stiuctural parameters;

- structures with large unit cells:

- disordered structures, with lattice-jas disorder, or with other defects:

- stepped surfaces, clean and adsorbate-covered:

- structures with incommensurate overlayers.

Wh shall look at the recently proposed approaches for dealing with these various forms of more complex and disordered surface structures. We shai limit ourselves to LEED and some of its closely-related electron diffraction techniques.

\section{Towards Diffraction from Complex and Disordered Surfaces}

\subsection{The Problem}

The central difficulty in extending LEED to more complex and disordered structures lies in the theory of multiple scatterinc. Multiple scattering leads to many possible scattering paths and to self-consistency requirements. The complications are the same as in solid-state bandstiucture and molecular-orbital problems. The issue is then to find suitable approximations.

Conventional LEED theory [1.4.5] has identified the necessary ingredients of a successful doscription of multiple scattering in relatively simple ordered structures. The most fundamental ingredients are the muffin-tin model and the description of electron scattering by atoms with the help of partial (i.e. spherical) waves. A partial-wave expansion of the scattered wave has to be truncated at a finite value $l_{\text {mas }}$ of the angular momentum $l$. This yields $\left(l_{\text {inas }}+1\right)^{2}$ partial waves at each atom. due to the allowed values of the magnetic quantum number $m$. The (complex) amplitudes of all these partial waves at all inequivalent surface atoms have to be determined in the process of simulating LEED intensities on a computer. Thus the number 
of atoms in the two-dimensional surface unit cell enters in the case of ordered surfaces: this unit call extends as deep into the surface as the electrons can penetrate. For disordered surfaces, the unit cell effectively becomes infinitely large and so does the number of inequivalent atorns $N$. However, many forms of disorder are in some way repetitive if the short-range order is considered (0.8. identical adsorption sites arranged without long-range order). so that the number of inequivalent atoms $N$ need not be large. We shall later discuss more thoroughiy why and how the long-range and short-range order can be separated for this purpose.

In Table 1 the cost of the conventional LEED computation is characterized in terms of its dependence on $I_{\text {mea }}$ and $N$ : the high power with which this cost rises clearly indicates where the problam lies. But the problem crops up in different ways as well. Wa may use the popular plane-wrve representation between atomic layers. Then, with ordered structures. the total number of diffracted beams comes into play. This number is equal to the number of plane waves used, and both are simply proportional to the two-dimensional unit cell area $A$. Table 1 shows how the computational cost scales with $A$ : depending on the calculational scheme used, a power law with a power between 2 and 3 is found. This again creates serious problems as the surface structure becomes complex. e.5. with large-unit-cell reconstructions or with large adsorbates.

With the plane-wave representation. the number of plang waves is also affected by the internuclear spacing between atomic layers: small spacings d require relatively many plane waves. As a rosult. the computational cost can rise. as Table 1 shows. with a strongly negative power of $d$. This is unfortunate, because the plane-wave representation would otherwise have been an attractive alternative to the sphericzl-weve representation.

Finally. we must consider the ener $y$-dependence of the computational cost of conventional LEED. With increasing energy $E$, the value of $I_{\text {mae }}$ increases slowly but surely. More seriously. the number of required plane waves increases rapidly. These two effects are combined in Table 1 to show a square-to-cubic dependence on $E$.

A different kind of difficulty with LEED for complex and disordered surfaces is the issue of finding an efficient procedure to determine the many unknown structural parameters. This problem is not unique to LEED. however. It exists equally well, for example, in standard $x$-ray crystallography. despite its relatively simple diffraction theory.

On the experimental side. two demands arise when dealing with complex or disordered surfaces. First, complexity requires an adequate data base to determine the additional unknown parameters. Thus. rapid and automated data acquisition is desirable. Second. for disordered structures the diffiuse LEED intensity needs to be measured. This leads to new approaches, which are already becoming available. We shall treat these experimental issues in Section 6.

\subsection{Basic LEED Methods}

Before entering into descriptions of the newly introduced theoretical techniques, it is necessary to state which basic ingredients these will use. We shall not get into the mathematical formalisms. but stay with a physical description of the processes that are included in the formalisms. More detailed treatments are available in the literature [1.4.5].

The basic ingredients to be introduced next are common to virtually all the LEED techniques. whether old or new. and should remain in force for the foreseeable future.

As mentioned earlier, the muffin-tin model is utilized to represent the scattering potential of the surface atomic lattice. It consists of spherically-symmetrical ion-core potentials, surrounded by a constant muffin-tin level. However, this muffin-tin level may change from one atomic layer to another. This happens of course in particular at the interface between lattice and vacuum.

A layered structure is often adopted in LEED to describe a surface. Atomic layers parallel to the surface are defined, in a way appropriate for each particular theoretical method. In a number of cases, a combined-space representation is chosen. which depends intimately on 
this layer approach. Namely, the wavefield is expanded in spherical waves within those layers. while it is expanded in plane waves in the gaps between those layers.

In the spherical-wave representation it is most common to use free-space Green functions to describe wave propagation from one atom to another. In this representation there are various ways of obtaining self-consistent solutions to the multiple-scattering problem. A "giant-matrix" inversion as proposed by Beeby does the job in a closed form. Then there are perturbation expansions, such as Reverse Scattering. which can converge to the same result. when multiple scattering is not too strong.

In the plane-wave representation many methods are available to treat multiple scattering. One is the Bloch-wave method with wave-matching across the surface. Another is layer doubling. Both of these have a closed form, with the second requiring sufficient damping of the wavefield into the bulk. A popular perturbation expansion of this problem is offered by renormalized forward scattering, in which the predominance of forward scattering is used to achieve rapid convergence (as long as multiple scattering is not too strong and damping not too weok).

We have mentioned damping: this is the effect of inelastic energy losses, which reduce the elastically surviving flux of electrons. The most common way of taking this in to account is through a small imaginary part of the scattering potential. It is usually assumed homozeneous and isotropic. Another equivalent approach is to introduce a mean free path length. that also exponentially dampens waves.

Finally, all LEED theories include Debye-Waller factors: these represent the effect of thermal vibration. namely the elastic or quasi-inelastic removal of electron flux into diffuse directions of difraction. At each scattering in a multiple-scattering chain a Debye-Waller factor is allowed to remove some electron flux.

The new methods introduced for complex and disordered surfaces use the same physical ingredients and many of the same calculational techniques described in this section. The difference often lies in a new packaging of multiple-scattering paths into units that are better adapted to the new tasks. Or some multiple-scattering paths are neglected. The end result either is equivalent to that of the old way, or is a suitable approximation to it.

\subsection{Theoretical Solutions}

We shall now describe general strategies that have been proposed to overcome the computational problems of LEED discussed in Section 2.1. We shall also briefly introduce specific methods. Some of these will be discussed individually in more detail in Sections 4 and 5.

\subsubsection{Cluster Methods}

With complex and disordered surfaces. plane waves lose some of their attractive features: their number increases rapidly with large unit calls, while their suitability becomes less obvious in the disordered case, since beams become diffuse. In addition, the long-range periodicity becomes a secondary aspect of the structure. in the sense that the immediate esvironment of each atom shows no sign of long-range order.

Thus, cluster methods have been proposed: in these the spherical-wave representation is used within clusters representing suitable neighborhoods of surface atoms. "Suitable" means: if one chooses a particular surface atom, the cluster should be large enough to include all significant multiple-scattering paths which pass through that particular surface atom. The finite electronic mean free path sees to it that the cluster radius can be made finite. for instance as small as perhaps $10 \mathrm{~A}$. As a consequence. one may have to choose a different cluster to represent the neighborhood of each inequivalent surface atom. This is schematically illustrated in Figure 5. In general, the clusters based on different atoms overlap each other.

The problem is hereby reduced to solving the multiple scattering within each cluster and 
then somehow combining the individual results. In this way, the computing time is made proportional to the number of translationally inequivalent surface atoms. a great improvement ove: their second or third power. But it does remain proportional to a high power of the number of atoms within each cluster.

Early cluster methods include those proposed by Duke et al [19], and by Moritz et al [20] for the calculation of diffuse intensities due to surface disorder. These methods were rather restricted in their applicability due to high computational costs. For example. the approach of Moritz et al could only handle very small clusters to represent the multiple-scattering effect of each atom's neighborhood. while the number of inequivalent clusters must remain small. But these approaches paved the way for future developments. They already included the concept of "kinematic cluster addition" (KCA), in which the total scattered wave is composed of kinematically added waves leaving inequivalent surface atoms after multiple scattering within each cluster.

A later development was the ono-center expansion [21], in which the spherical-wave scattering properties of an entire cluster were obtained self-consistently and then were available for calculating plane-wave diffraction in many situations. The approach was made efficient by breaking down the clusters into concentric shells and separating the problem of the scattering by individual shells from that of the scatterine by the assembly of shells [22.23]. A variant of this method was developed for steps. or. more generally. for "linear defects" [24a]. Here. cylindrical waves mere used to describe the multiple scattering within individual chains of atoms. which are chosen paralled to the linear defect. These chains were then combined into three-dimensional surfaces (this mothod has close analogies with the earlier "chain method" developed for medium - and high-nnergy' electron diffraction [25]). Another cluster method was proposed by Marcus et al [26]. These methods exhibit the common feature that the computing time scales in simple proportion to the number of inequivalent surface atoms.

\subsubsection{Reducing Multiple-Scattering Paths}

Another strategy starts from the observation that the computational cos: is directly due to the large number of multiple-ecattering paths that need to be calculated. Perhaps one could identify and iznore large classes of such paths that contribute only weakly to the diffracted intensities. In dense motal surfaces, for which the conventional LEED theory was developed. it is difficult to find classes of weak paths.

But in many other materials. multiple scattering is much less important. For example. aluminum and silicon are more "kinematical" materials as far as LEED is concerned. And so are many other open-lattice semiconductors. as well as many low-density materials and overlayers. especially molecular overlayers.

Two factors reduce multiple scattering: a low packing of the material and a light nuclear weight. A low packing means larger interatomic distances on the average. which reduces the number of possible multiplo-seattering paths, given a constant mean free path. A light nuclear weight reduces the amplitude of any seattered wave. especially of a multiple-scattered wave.

Weak multiple-scattering paths can bo eliminated in several ways. First, one may simply shrink the size of the above-mentioned clusters. within which one allows multiple scattering around each atom. In this manner, the individual cluster calculation is made much faster. This approach already had to bo followed by Moritz et al [20]. It stands the best chance of being reasonably accurate in loosely-packed materials. whore the average distance between atoms and their various shelis of neighbors is ralatively large. Thus, semiconductors and molecular materials are good candidates for this treatment.

It is also possible to prevent long distances between successive scatterings in a multiplescattering path: far fewer paths are then allowed. Thus. in the method called - near-neighbor multiple seattering" (NNMS) [27], a wave is only allowed to travel unscattered from a given atom to its nearest neighbors. In other words, a multiple-scattering path is only allowed to consist of short "hops" between nearest neighbors (but a string of short hops may lead far 
away). There may be as many hops as the mean free path allows. This approach does not reduce the scaling power of the computing time dependence on $N$, the number of atoms in a unit cell or cluster, but it does reduce its prefactor considerably.

\subsubsection{Kinematic Sublayer Addition}

A simple but very effective variant is " kinematic sublayer addition" (KSLA) [28]. It applies to the case where the clusters are disjoint, i.e. no multiple-scattering path can hop from one sluster to another. An example is molecular layers, where the hop from an atom in one molecule to an atom in another molecule is often too large to provide a significant contribution to intensities. In that case the scattering properties of the separate molecules can be calculated independently. They can then be kinematically combined and recombined for many different relative positions of the different molecules.

\subsubsection{Forward Focusing}

One may also exploit the wel-known predominance of forward scattering of electrons by atoms. The idea is to neglect paths that include many "sharp bends". An old implementation of this approach is the "quasi-dynamical" theory [29,5], in which intra-layer multiple scattering is calculated kinematically. while interlayer multiple scattering is calculated more exactly.

This kind of approach is especially useful at higher energies, above about $200 \mathrm{eV}$. where forward scattering is so pronounced as to become forward focusing. A cluster-oriented method that specifically exploits forward focusing is called "near-field expansion in clusters" (NFEC) [30]. Here the wavefield scattered by an atom is expanded about a noarby atom center most often in the forward direction. using a "Taylor series. magnetic quantum number expansion" (TS-MQNE) [31]. This replaces the conventional accurate representation of the scattered wavefield in all directions around the scattering atom. The resulting computational gain is considerable. The cost becomes proportional to the square root of the energy and directly proportional to the number of inequivalent atoms. And at higher energies more experimental data can be eathered, as a larter part of reciprocal space is accessible.

\subsubsection{Beam Set Neglect}

Next we consider a method that is plane-wave oriented. Thus, the intention is to identify and eliminate sets of plane waves that do not contribute significantly. In this case, one holds on to the plane-wave representation as much as possible, but simplifies it to bring its escalating

cost under control. Such methods often combine well with cluster approaches. cumulating the benefits.

The "beam set neglect" (BSN) method [32] applies to uverlayers or reconstructed layers with a superlattice of a disordered lattice on a perfect substrate. it recognizes the fact that only a very limited set of plane waves (beams) contribute signifieantly to the detected intensities: many sets of beams can be neglected in the calculation. As a result, the dependence of the computation cost on the unit-cell area $A$ and the energy $E$ fall from the second or third power to no dependence. In other words, the computation cost can be made independent of the enerty. the unit-call area or the presence of disorder. This approach works also for incommensurate overlayers. BSN can be very effectively combined with cluster methods, in particular the KSLA method.

\subsubsection{Tensor LEED}

A third basic approach is not to give up multiple-scattering paths. but to approximate their contributions as being linear expansions from a nearby surface geometry that was treated 
exactly. In other words, one would compute exactly the LEED intensities for a given reference structure. preferably a simple one: for example, a highly-symmetrical or undistorted structure. Then, the intensities for a somewhat deviating structure would be computed, using a linear expansion in terms of the structural parameters. This yields excellent computation times. especially if many deviating structures around the reference structure are explored. because the linear expansion itself is a very simple operation. This is the philosophy of tensor LEED [33]. which has already been applied to periodic surface reconstructions and to disordered overlayers that induce substrate distortions.

\subsubsection{Combinations}

It should be pointed out that many of the techniques described above can be combined to cumulate their advantages. This is especially so because in LEED one often decomposes a surface into layers parallel to the surface. Each layer can be defined and treated with a method that best suits its structure: often this will be a spherical-wave and/or cluster method. And between layers one may apply plane waves and choose approximations accordingly. This gives great latitude for finding efficient, although case-dependent. methods to solve complex and disordered surface structures.

If one uses approximate methods as proposed above, one stands the obvious risk of obtaining incorrect structural results. For that reason. sequential approach can be used: one riay start a structural search with a broad analysis of many possible structures using an approximate and efficient computational method. In this way many unpromising structures can be rapidly eliminated from consideration. The next step is to concentrate on the relatively fow remaining promising structures and to test them with a more accurate LEED theory. The process can be repeated with increasing accuracy. yielding not only a (hopefully) unique structural solution, but at the same time a sefined structure.

\section{Order vs. Disorder and Diffraction}

It will be very helpful for our further discussions to analyze more clearly how order and disorder affect the diffraction of electrons by a surface. This is rather well understood for kinematic diffraction (e.g. in $x$-roy diffraction). but often leads to some confusion in LEED due to multiple scattering.

Figure 6 summarizes a fundamental distinction that has to be made here. It illustrates the case of an ordered surface. Such a surface is characterized by a "lattice" and a "basis". The lattice describes the periodicity of the surface, i.e. the shape, size and orientation of the unit cell, but not its contents. Thus, the lattice describes only the long-range structure. It is responsible for the presence of sharp LEED beams. which correspond to the reciprocal lattice of the surface lattice, as given by the Brars diffraction conditions. It is important to realize that. althourh these are purely kinematic considerations, they ara not affected by the presence of multiple seattering. Indeed, the LEED spot pattern can be very accurately predicted with the kinematic Brars conditions. We shall call this lattice-induced contribution the "structure factor", in close analogy with x-ray terminology.

The situation is different with ihe "basis". The basis is the set of atoms that is contained within any unit cell. together with their individual positions and scattering properties. The basis therefore relates primarily to the short-range order. The intensities of the LEED beams (rather than their existence and direction) is governed by the basis. These intensities are strongly affected by multiple scattering. They depend in particular on the relative positions of the basis aroms, not only throuch the kinematic phase factors, but also very much through the multiple scattering. Therefore, intensities are often said to be "dynamical" and this is of course the origin of the label "dynamical LEED". We shall call this basis-induced contribution 
the "form factor".

Thus, the diffraction pattern is primarily determined by long-range order, while the diffraction intensity is primarily determined by short-range order. In fact, one may view the observed LEED to be the product of the smoothly varying basis-induced form factor and the sharply spiked lattice-induced structure factor.

This realization is central to understanding diffuse LEED due to disordered surfaces. Let us take an ordered surface and gradually disorder it in such a way that the short-range order is not changed appreciably. This is most readily visualized with a low-coverage overlayer that becomes disordered without changing the individual adsorbate sites (so-called lattice-gas disorder). Then the multiple-scattering is essentially unchanged, because the neighborhood of each atom is largely unchanged. What matters here is the neighborhood within, say, one mean free path length. Thus the smooth "basis-induced" form factor is almost unchanged from the ordered case. However, the loss of long-range order has a profound effect on the "lattice-induced" kinematic part of the problem: there are no Brags conditions any more, but instead diffraction may occur in any direction. The structure factor and therefore the LEED pattern become diffuse (however, there may still be sharp spots, due solely to the still-ordered substrate).

Now again, the final pattern can be viewed as the product of a kinematic structure factor reflecting the long-range disorder and a dynamical form factor reflecting the local short-range order. However, it is no longer easy to distinguish these two contributions. The structure factor of the disordered part of the surface no longer stands out as sharp spots. But an I- $V$ curve, which shows the intensity measured as a function of electron energy for constant momentum transfer parallel to the surface, will look very rruch like that of a sharp spot: most of the structure of such an I-V curve is due to the short-range order, whether the surface is ordered or disordered. This is because a constant momentum transfer parallel to the surface keeps the structure factor constant in this measurement (at least if there is purely twodimensional disorder only). A more mathematical treatment of these questions is available elsewhere [34].

\section{Cluster-Oriented Approaches}

We shall now discuss in some more detail a few modern LEED methods that are based on clusters. They involve primarily the spherical-wave representation. Here again. we shall refrain from showing detailed formalisms and concentrate on their physical meaning.

\subsection{Kinematic Cluster Addition}

Let us consider the electrons that are detected after the diffraction process from a surface. They are represented by a wave that has left the surface. It is easy to imagine this wave to be composed of individual waves that have left aach atom of the surface. Let us choose one such wave having left a particular atom. This wave includes all possible ways for electrons to travel from the source (electron gun) into the surface, via any number of scatterings. until final scattering by that particular atom. In other words, we have classified all possible multiplo-scattering paths according to which is the last scattering atom in these paths. Figure 5 illustrates the situation: waves with amplitudes $F_{i}$ and $F_{j}$ leave atoms $i$ and $j$, respectively. They are built up of all possible scattering paths that end up in atoms $i$ and $j$. respectively.

Since we have decomposed the final wave into a superposition of waves coming from different surface atoms, the task is now reduced to calculating the waves coming from each surface atom separately. Then. their superposition is a kinematic addition of wave amplitudes. i.e. an addition of amplitudes that takes into account the phases of the different waves due the relative positions of the last scattering atoms. Such an addition of amplitudes is the same 
as that practiced in kinematic $x$-ray diffraction, for example. The difference is that in $x-r a y$ diffraction the wave amplitudes are simply given by the atomic scattering amplitudes, while in LEED they are much more complicated due to multiple scattering before arrival at each surface atom.

The significance of this approach is that the computational problem has been made proportional to the number of inequivalent atoms at the surface, rather than scaling with a high power of that number. (We say "inequivalent" atoms, because if two atoms have identical surroundings, the maves leaving those two atorns towards the detector will be identical, except for the simple kinematic phase factor.)

We are however left with the significant problem of calculating the individual waves leaving the inequivalent surface atoms. This is where the cluster concept enters. We choose a cluster of finite size around such a surface atom, with a radius comparable to the mean free path. We then allow the incident electron to travel in any way that it wishes through that cluster up to the chosen surface atom. The result is the desired wave amplitude leaving that surface atom.

Practical methods of solving this multiplo-scattering problem in a cluster have been slow in coming. Perhaps the most attractive at present is that of Pendry and co-workers [22,23]. They propose that a cluster be decomposed into concentric shells of atoms around the surface atom of interest. First the scattering properties for each shell taken separately are calculated. This is done in the spherical-wave representation to take advantage of the spherical geometry. The incoming electron wave is also decomposed into spherical waves centered on the surface atom in question. The spherical waves are then allowed to propagate inward from shell to shell (including any number of back-reffections between shells) until they arrive at the central atom. The result of the last scattering by the central atom is the desired wave amplitude. This approach is analogous to the layer-by-layer approach of layer doubling or renormalized forward scattering in the plane-wave representation: the role of the layers is now played by the shells.

Note that the method has made no assumption about long-range order: it applies equally well to ordered and disordered surfaces. In fact. one may treat many kinds of surface with this approach. Any form of point defect can be handled. such as an adatom. a vacancy. a substitution, an interstitial, including local distortions caused by such defects. Ordered defects represent ordered overlayers. reconstructions, alloys, etc. Disordered defects represent: random adsorbates, impurities, distortions, atc.

The method has been applied to the solution of the adsorption structure of disordered oxygen on $W\{100\}$ [6]. Not only was the oxygen position determined from the diffuse intensities, but more recently a significant oxygen-induced distortion of the surrounding $W$ lattice wis found [33]: the oxygen atoms occupy fourfold hollow sites of the metal substrate, and they pull the four surrounding $W$ atoms inward, reducing their mutual $W-W$ distances.

An approximation to the cluster method is achieved by restricting hops in a multiplescattering path to only nearest neighbors. Figure 7 illustrates what kind of path is accepted and what kind is not. This " near-neighbor multiple scattering" scheme [27] severely reduces the number of possible paths and thereby reduces the computational effort. As mentioned in Section 2.3. this approach reduces the prefactor of the computing time, rather than the power law of its dependence on the number of atoms in the unit cell or in the cluster. It therefore does not in itself make a big difference, but does help other methods perform somewhat: faster.

\subsection{Near-Field Expansion in Clusters}

We here describe a method [30] that utilizes the fact that forward scattering of electrons by atoms dominates over scattering in other directions. This "near-field expansion in clusters" (NFEC) stems from a method developed for angle-resolved photoelectron diffraction at surfaces: ARPEFS ("angle-resolved photoelectron fine structure"). It was designed t-r slightly higher energies than is customary in LEED. but can be used down to LEED energies.

Forward scattering has long been known to be important in LEED, especially towards 
higher energies $(>100 \mathrm{eV})$. In fact, it was the basis for the renormalized forward scattering method. However, all LEED methods so far have used an exact description of the scattered wave not only in the forward direction, but all around the scattering atom. This is a waste of effort if most scatterings occur in the forward direction. The NFEC method therefore performs an expansion of the scattered wave about the next atom that will receive it "downstream" (the procedure is repeated if there ars other atoms downstream of a given scatterer). The expansion is done in two dimensions: radially and azimuthally. By choosing suitable local coordinates for each scattering, the expansion has few terms. resulting in very efficient computation.

This is a cluster method, because the scatterings from atom to atom are followed up individually in chains of multiple scatterings that are restricted to a cluster. The shape and size of the cluster is governed by the mean free path and by the cone formed by the forward scattering. The algorithm continuously adjusts that shape and size as it procends from scattering to scattering.

Being a vluster method, the computation cost is simply proportional to the number of inequivalent atoms in the surface. In terms of energy dependence. NFEC scales as the square root of the energy. Being a spherical-wave method, its performance does not depend on the unit cell size or on the distances between atomic layers. And it scales oniy linearly with the number of partial waves used, $I_{\text {mas. }}$.

NFEC has been applied to reproduce and explain in more physical detail than possible so far the LEED intensities from simple surfaces. And it has behaved as efficiently as expected for larger energies. providing a very attractive way to calculate medium-energy electron diffraction intensities. It can now also be used to attack complex and disordered surfaces.

A variant of NFEC has been developed [35.36] to study the emission of electrons from surfaces by Auger and other inelastic processes at the higher energies, say $500-1000 \mathrm{eV}$. This is directly analogous to angle-resolved photoelectron emission: in some manner an electron of a given energy is created within the surface; it then elastically travels out. undergoing multiple scattering in the process; the resulting emitted electron distribution reflects the surface structure and can be used to obtain it. Relative to the LEED problem, there are two main differences here. First. the electrons of interest are deemed to start their useful life at some atomic location within the surface, rather than at an electron gun outside the surface. Second. the electron "creation" is an incoherent event. unselated in its phase to any other electron creation elsewhere.

Corresponding full multiple-scattering computations have been performed for the first time and match experimental data well [36]. For instance, Auger electron emission datá were analyzed for monolayers of $\mathrm{Cu}$ embedded within a Ni $(100\}$ surface: the depth at which the $\mathrm{Cu}$ layers were embedded could be determined. Also. inelastic "Kikuchi-like" electron emission was reproduced. which allowed the site determination of oxygen adsorbed on $\mathrm{Mg}\{0001\}$ : the oxygen atoms were found to be preferentially buried interstitially in octahedral sites below the second metal layer.

An interesting feature of this electron emission is that forward-focusing peaks are observed experimentally: along crystallographic chains of atoms one systematically finds an enhancement in electron emission, within a cone of perhaps $10^{\circ}$ half width. The reason for this is that a spherical wave leaving one atom is focused behind each neighbaring atom as if the latter acted like a converging lens. This effect was first observed and explained by Egelhoff in the case of photoemission and, later. Auger electrons [37].

However, the NFEC calculations [36] clearly show that other peaks not related to interatomic directions exist as well. These are due to interference effects between different paths leaving the surface. Thus, one has to be careful not to interpret every peak as an interatomic direction: a full calculation is normally required to extricate these two kinds of peaks due to focusing vs. interference.

\section{Beam-Oriented Approaches}




\subsection{Plane Waves Despite Large Unit Cells and Disorder}

Despite the advantages of spherical waves for treating diffraction by complex and disordered surfaces. plane waves retain a great attraction. They are mathematically always far easier to treat and lead to very efficient computation whenever they can be used. This has been recognized a long time ago in the case of electron diffraction at surfaces. For example. photoelectron emission has been treated with plann waves for over a decade, even though there are no naturally obvious plane waves in the probiem. As mentioned in the last section. such olectrons start out as spherical waves emitted by some atom and then propagaie via multiple collisions out from the surface.

However, the detector by its very presence and narrow angular aperture defines is clear diraction: from surface to detector. This direction in turn singles out a plane-wave component of the emitted spherical wave. while the other plane-wave components travel undetected past the detector. Thus a well-defined plane wave can be chosen. It can then be traced back toward the surface and. in reverse time. its diffraction by surface layers can be followed. If a periodical surface layer is encountered, the diffraction leads to a complete set of plane waves that correspond to the two-dimensional reciprcsal lattice of that layer. This is directly analogous to the plane-wave diffraction process in standard LEED. where a periodic suriace generates a set of well-defined diffracted beams. In the time-reversed thinking process. one can follow up the waves back to their source. This might be an electron gun. in the LEED case. It could also be a photoemission or Auger electron or other electron emission process.

In the particular case of LEED from disordered surfaces, there is a diffuse diffraction process to be included. Incoming electrons may diffract as plane waves from perfectly periodical layers, but as soon as a defoct is encountered, any plane wave scatters into spherical waves. However. we are again only interested in those electrons that will travel in the one direction in which the detector is located. Thus. again plane waves are defined and the calculation can focus on those and ignore other scattered waves.

The general picture we get is the following. If there is an electron gun in the problem, it defines an incident plane wave. together with scattered plane waves related to it by the surface reciprocal lattice. And if there is an angle-resolving detector as well. it defines an emitted plane wave, together with other plane waves relate: to it by the saine surface recipracal lattice. Thus we obtain two sets of important plane waves:. These can be selected out. while any other plane waves can be neglected. One does pay a price for this in the case of large-unit-cell or disordered overlayers. Some plane waves with non-vanishing amplitude are neglected in this process. We shafl discuss below for individual techniques whether the results are adversely affected.

\subsection{Beam Set Neglect}

As discussed earlier, there is great benefit in reducing the number of plane waves that a calculation needs. This is because all the plane waves consistent with the periodicity of the surface are interconnected by the multiple scattering: as a resuit. all the plane-wave amplitudes must in principle be computed simultaneously in a self-consistent manner, ar:d thus large calculations result when there are many beams.

In the layer treatment of surfaces. we would compute the diffraction properties of the overlayer and of the substrate taken separately. These diffraction properties would be obtained for all the beams (plane waves) consistent with the periodicity: in the ordered case, the beams are all the superiattice-implied beams: in the disordeped-overlayer case. one would have to include the infinite set of all possible plane waves, an impractical proposition. Then, with layerstacking techniques such as renormalized forward scattering or layer doubling, the overlayer would be added onto the substrate. yielding the final beam intensities.

\subsubsection{Two Important Beam Sets}


The beam-set-neglect method simpiifies this problem as follows. Let us focus our attention on a particular detected plane wave and ask for its intensity at the detectcr. According to Section 5.1. two sets of plane waves are then particularly important. We shail define those two sots more accurately now for our present purposes [32]:

1. The set of plane waves $\mathbf{0}+\{\mathbf{G}\}$, where $\mathbf{0}$ represents the incident plane wave and $\{\mathbf{G}\}$ represents all the two-dimensional reciprocal-lattice vectors of the substrate: this defines the "integral-order beams" and we shall call them the "incident set". It is illustrated in Figure 8 as the beams $k_{i n}+g_{1 \times 1}$, to stress the role of the substrate periodicity.

2. The set of plane waves $k_{f}+\{G\}$, where $k_{f}$ represents the final detected plane wave and $\{G\}$ again represents all the two-dimensional reciprocal-lattice vectors of the substrate; this defines a shifted copy of the "integral-order beams", such that one of them goes straight to the detector: this set can be called the "detected set". Figure 8 shows this set as the beams $k_{\text {out }}+g_{1 \times 1}$. again stressing the role of the substrate periodicity.

The reason for the choice of the substrate vectors $\{\boldsymbol{G}\}$ is this: the substrate lattice can only diffract a beam by adding a vector $\mathbf{G}$ to its parallel component. This means that the substrate cannot diffract a beam out of set 1 into set 2 or any other such shifted set of beams. Only the overlayer. whether ordered or disordered, can diffract out of these two beam sets. As we shall see below. the result is that only higher-order multiple-scattering terms are excluded by restricting ourselves to these two beam sets.

If we are dealing with an ordered overlayer, the detected set corresponds to choosing a particular "fractionai-order beam" $k_{j}=\mathbf{g}$. and including all the beams obtained from it by the substrate reciprocal lattice: $\mathbf{g}+\{\mathbf{G}\}$. Thus, if there are $;=$ "integral-order beams" included in the calculation, the calculation would use $2 n$ plane waves. Let us compare this with the conventional LEED calculation. If the overlayer has a unit cell with an area $F$ times larger than the substrate unit cell, then the conventional approach would include $F n$ rather than $2 n$ plane waves. When $F$ is large, this makes a big difference, since it is the second $c r$ third power of the number of beams that comes into play: we obtain a reduction in computational effort by a factor of about $(2 / F)^{2}$ or better, which can easily be one or more orders of magritude. For example, with a $(3 \times 3)$ overlayer, the reduction factor is $(2 / 9)^{2} \simeq 0.05$ or better.

However. the 2 -beam calculation only computes the intensities for those $2 n$ beams. since all the others have been excluded. In many cases. this subset of $2 n$ beams may already be sufficient to do a structural determination: then the beam-set neglect approach has delivered a result at a computaticnal cost which does not depend on $F$. the unit cell size.

For more accuracy, and especially when the structure is crimplex and involves many unknown parameters, one may wish to use more than $2 \mathrm{n}$ beams in the comparison with experiment. This can be handled quite simply by adding more computations similar to the one just described: one chooses a new detected beam that was not already computed and makes a new computation with the new pair of beam sets based on the incident beam and this new detected beam. This produces the intensities of $2 n$ beams, $n$ of which are duplicates of the previously calculated integral-order beams. That leaves $n$ new intensities, giving $3 n$ overall so far. This can be repeated for other emitted directions, such that each additional computation adds $n$ more intensities. One can ever go all the way to exhaust all $F$ sets of beams, requiring a total of $F-1$ computations. Thus, even in this worst case. the total computational cost is only directly proportional to the area of the unit cell, rather than to its second or third power.

Other combinations $c$ beam sets can be used to enhance the accuracy of the cornputation. but at a cost in computational effort. For example. there is no need to neglect all but two beam sets. One might include more than two such beam sets at once in the rame computation. This reintroduces a number of multiple-scattering pathways and thereby enhances the accuracy. In the limit of inclusion of all beam sets in a single calculation, one simply recovers the full conventional LEED formalism. Thus the accuracy of the beam-set-neglect approach can be tailored to one's needs.

This is illustrated by Figure 9, in which different levels of accuracy are applied to the calculation of $I-V$ curves for an overlayer of benzene molecules on a Rh\{111\} substrate, with 
a periodicity of $(3 \times 3)$. The curves labeiled $(3 \times 3)$ are calculated using all beams, i.e. without beam sot neglect (but without multiple scattering within the molecular layer, which however is not relevant to the comparisons made here). The integral-order beams (10) and (01) also show curves labeled $(1 \times 1)$. When the detected direction coincides with one of the integralorder beams due to the incident beam, then the two sets 1 and 2 defined above are identical to each other. Therefore, the two beam sets collapse into just the set $\mathbf{0}+\{\mathbf{G}\}$, i.e. the set of waves that the clean surface would produce. However, their intensities are much different from the clean-surface intensities, alsc shown in Figure 9. It can be seen how well beam set neglect reproduces the "exact" (3x3) curves of the $(10)$ and $(01)$ beams.

Two "fractional-order" beams are shown in Figure 9. The intensities of one of them has been approximated by using the " $(3 \times 1)$ beams". This is a particular group of three sets of beams, rather than the two sets of the standard beam-set-neglect method. Again one sees very good agreement in the structure of the $1-V$ curves. especially in the positions of peaks and valleys. These are the most important features of $I-V$ curves for structural determination. The other fractional-order beam is also treated with a group of three (other) sets of beams. and the same comments apply to it.

\subsubsection{Accuracy of Beam Set Neglect}

We must next discuss in more detail the question of the accuracy of beam set neglect relative to the "exact" conventional calculation, which does not neglect plane waves. To that end. we can ask which multiple-scattering paths are ignored due to team set neglect. The answer is simple: only scattering paths with three or more scatterings are ignored (none of these scatterings being in the forward direction). And, since three or more scatterings (other than purely in the forward direction) give weak contributions. we are only ignoring weak correctiors to tic final intensity.

That we are only ignoring paths with three or more scatterings can be easily seen as follows. The neglect of a certain beam $y$ not included in the two sets defined above prevents multip:-scattering paths of the kind; scattering from the incident beam 0 to beam $\mathbf{g}$ by the overlayer, then reflection from $g$ to $g$ by the substrate, and finally scattering from $g$ to $g$ by the overlayer, where $\boldsymbol{g}$ is the final detected beam. Thus. three scatterings are needed in this path. More generally, at least three scatterings will be found in any path that includes an intermediate beam of the excluded type $z$.

Thus, whenever multiple scattering is not too strong. beam set neglect should work well. This favors systems where the scatterers in either the overlayer or the substrate are not too strong. e.g. organic overlayers on metallic substrates. Figure 10 illustrates the accuracy of beam set neglect in a practical situation: a layer of benzene molecules lying on a $R h\{111\}$ surface with a $c(2 \sqrt{3} \times 4)$ rect periodicity [32]. The figure quantifies the agreement between theory and experiment for two different hollow adsorption sites. and as a function of the spacing between the top $R \mathbf{h}$ nuclear plane and the $C_{\theta}$ nuclear plane. The comparison with experiment is made both without and with beam set neglect. The differences between the two cases are seen to be very small. and both clearly favor the same geometry: the "bABC hollow" site with indistinguishable layer spacings. (The multiple scattering within the molecules was in this case calculated using the near-neighbor multiple scattering method.)

\subsubsection{Surface Reconstruction}

The beam-set-neglect method can be applied to surface reconstructions as well as overlayers. One then merely considers the reconstructed layer to constitute one overlayer. More generally, beam set neglect assumes that the surface consists of a substrate that supports an overlayer which has a superlattice or disorder. The method exploits the contrast between the substrate lattice and the overlayer lattice. This, however, implies that the method has no advantage in the case of a very thick overlayer or reconstruction, since then the electrons do 
not reach the substrate and the method cannot benefit from its different lattice.

\subsubsection{Disorder}

2He have not much differentiated between ordered and disordered surfaces in the discussion so far about beam set neglect. Indeed, there is little difference in the theoretical justification of the method for these two cases. For diffuse intensity calculations, one has tine option of abtaining an I-V curve for the emitted direction. or a two-dimensional distribution of intensity across the screen.

In the former case. our reasoning [34] and theoretical tests indicate that one gets the same $1-V$ curve appearance as if the surface were ordered. In other words, to measure the $1-V$ curve of a spot due to an ordered surface or of the disorder-induced diffuse intensity in the same position as the spot gives the same result. This confirms that it is the local short-range that gives rise to the peak-and-valley structure of $1-V$ curves. However, one must pay attention to the fact that traditional spot $\mid-V$ curves are measured by following the spot as it moves across the screen with energy. This keeps the parallel momentum transfer constant. The corresponding $1-Y$ measurentent of diffuse intensities must alss maintain a constant parallel momentum transfer, i.e. track the position where a spot mouid be if the surface were ordered.

A similar word of caution applies to the measurement of the two-dimensional distribution of intensity at a given energy. A typical distribution whei ws have calculated with beam set neglect is presented in Figure 11. One often will need the logarithmic derivative of the intensity with respect to the energy in order to eliminate the effect of the long-range structure factor (see Sect. 6). This is based on the independence of the structure factor from the paralle! momentum transfer (at least for two-dimensional disorder). The implication is that the logarithmic derivative of the intensity with respect to energy should be measured at constant parallel momentum transfer, i.e. as if one were tracking a spot position [34.38].

\subsubsection{Incommensurate Oveplayers}

Beam set neglect has provided a becter solution to the problem of calculating diffracteo intensities from incommensurate overlayers [39]. By incommensurate we mean that the overlayer has a two-dimensional lateice which is independene of that of the substrate. This situation is common with overlayers that are strongly cohesive and can ignore the periodicity of the substrate on which they lie: for instance, graphite and oxides or other strong compounds form overlayers with their own bulk lattice, which in general does not match that of the substrate.

By using exactly the same arguments as above for ordered and disordered overlayers, one can easily show that acceptably accurate calculations can be performed with just the two sets of beams defined earlier. Again, the effect is to ignore weak third- and higher-order multiple-scattering paths. This approach has been applied to the structure determination of a graphite layer grown from hydrocarbon decomposition on a Pt $\{111]$ substrate [39]. The result is illustrated in Figure 12 . It was round that a single graphite layer rests on an incomplete layer of individual carbon atoms chemisorbed in hollow sites on the Pt substrate. The interlayer distances found are very reasonable when compared with known $\mathrm{Pt}-\mathrm{C}$ bonds and with Van der Waals dimensions for graphite.

\subsubsection{Combination with Other Methods}

Finally. it must be pointed. out that beam set neglect can be very efficiently combined with the other approaches described in this text. This is because beam set neglect does not prescribe how the scattering of the overlayer or of the substrate are to be calculated. These can be obtained with any other suitable method. For instance. beam set neglect can be combined with cluster methods. in particular with kinematic sublayer addition (see Sect. 5.3). 
This combination was in fact used by us to analyze the structures of benzene and coadsorbed CO deposited on Rh [17.28]. Pt [40] and $\mathrm{Pd}\{111\}$ [18]. In each case the computation was efficient enough to allow 1000 to 2000 structures to be investigated.

\subsubsection{Advantages of BSN}

Let us summarize here the advantages of beam set neglsct (BSN):

- BSN is applicable to any superlattice, including low-density adsorbate structures. large adsorbates, many adsorbates per unit cell (e.g. regular out-of-phase domains) and complex surface reconstructions:

- BSN allows higher energies, since fewer beams are used, where more experimental data are accessible;

- BSN allews calculating intensities for only a small number of beams. permitting efficient elimination oi many incorrect structural models:

- Many structural parameters can be optimized in a preliminary fashion with BSN at low cost; [2.5]:

BSN is easily programmed as a natural extension of the existing Combined Space Method

- BSN applies to disordered and to incommensurate overlayers.

Disadvantages of BSN are:

- BSN assumes that the overlayer is thin enough to let the electrons reach the substrate. which in addition must have a different two-dimensional lattice from that of the overiayer:

- Since approximations are involved in BSN. structural results are less accurate than with a full calculation (in our experience, the inaccuracies are often smaller than those already inherent in the LEED method):

- To benefit from linearity of the computation time with the number of inequivalent atoms. it is necessary that multiple scattering not take place over large distances, i.e. the inelastic mean free path should be small compared to. say, the unit cell dimensions.

\subsection{Kinematic Sublayer Addition}

We consider here a particular cluster method that simplifies into a very efficient plano-wave method. Kinematic sublayer addition [28] is soncerned with slusters that are well separated. The most obvious example is a rnolecular layer, where each molecule defines a cluster. Within each cluster multiple scattering can be calculated with any desired accuracy. But we neglect the direct scattering of an electron from one molecule to any other molecule. The greatest advantage of this approach comes when one deals with more than one molecule pe: unit cell in the ordered case, and with two or more inequivalent adsorbates in a disordered overlayer.

The specification "direct scattering" refers to the following assumption. which in turn is based on the use of plane waves. We treat the layer of clusters (e.g. molecules) as a separate overlayer, whose plane-wave diffraction properties are to be calculated in the absence of the substrate. Then, we combine the overlayer with the substrate by using any of the plane-wave layer-stacking techniques, e.g. renormalized forward scattering or layer doubling. This layerstacking step introduces multiple scattering between the overlayer and the substrate. As a result, it remains possible for electrons to first scatter from an adsorbed molecule. then be reflected from the substrate and finally scatter from another adsorbed molecule. Thus indirect scattering between clusters is allowed via the substrate, even if direct scattering is not.

The plane waves that are to be used in this layer-stacking process depend on the application. If one deals with an ordered overlayer, one may use the complete set of plane waves defined by the overlayer periodicity. However, it is also possible in the same case to use beam set neglect, i.e. to limit the plane waves to the "incident" and "detected" sets: the incident plane wave together with the substrate-diffracted plane waves, and the final detected plane 
wave of interest together with those plane waves which are scattered into it by the substrate. This process is to be partially repeated to treat other emission directions.

The advantoges of this approach are clear. First, the multiple-scattering within the individual clusters can be precomputed. stored and then used in many geometrical positions of the clusters relative to each other and relative to the substrate. This is because the positioning of the overlayer and its components is very efficiently done in the plane-wave representation. and thus can be repeated many times at little extra cost.

Second, it is almost no extra effort to include a higher density of the same molecules in - molecular overlayer: the additional molecules occur as further terms in a simple kinematic sum over the molecules contained in the layer. And the extra molecules need not occupy the same adsorption sites as the firat ones (but they should have the same orientation. of else additional calculations are needed. which are proportional to the number of orientations).

Third, the method can be equally well used for ordered and disordered overlayers.

Fourth, one may just as well mix molecules with individual adatoms, rather than with other molecules. For that matter, it is also possible to mix different adatoms in this way.

The question is, however. whether one obtains a reasonable approximation by ignoring direct scattering between molecules. The answer depends simply on how closely the molecules are packed together in an overlayer. If the molecules are very tightly packed. there is a bigger chance of an electron scattering from ona molecule to a neighboring molecule. Figure 13 shows the geometry. Around each adsorbate a sphere is drawn. within which multiple seattering is assumed to be important. When one sphere includes anoth:ar adsorbate ("overlapping spheres"), scattering from one adsorbate to the other can be important and affect the result.

We have made test calculations for one of the worst cases (27b): closest packing of $\mathrm{CO}$ in an overlayer. This is the case of a $(2 \times 2)$ structure of $\mathrm{CO}$ formed on $\mathrm{Rh}\{111\}$. which has a coverage of 0.75 molecules per surface metal atom. The result was that the optimized structural parameters, such as bond lengths, for this system were less than $0.1 \AA$ off when kinematic soblayer addition was used. relative to the case of full multiple-scattering calculations. In this particular worst case, one would need a better approximation for fine tuning of the structural parameters.

On the other hand, wave performed the same tests for overlayers involving hydrocarbonr. Since hydrozen is a very weak scatterer, the hydrogens form "cushions" between the different molecules. The space occupied by the hydrocens produces no detectable diffraction and thus effectively separates the scatterers in the different molecules by $5 \AA$ or more. Multiple scattering is quire negligible between such light atoms as carbon. nitrogen and oxygen over these distances. Thus, one obtains very good structural results in such cases. We have tested this in particular for benzene overlayers cosadsorbed with $\mathrm{CO}$.

We have extensively applied kinematic sublayer addition to the structural determination of a number of molecular coadsorption structures or metal substrates. These include benzene coadsorbed with CO. two examples of which are i.:ustrated in Figures 2 and 3 [17.18.28.40]. ethylidyne coadsorbed with CO or NO [41], and CO coadsorbed with Na [42].

\section{Experimental Requirements}

On the whole, the experimental techniques have needed relatively less evolution than did theory to attack complex and disordered surfaces. For complex ordered structures with many diffraction spots, it is mostly a matter of more conveniently acquiring the larger amount of data. This favors automated systems, for instance video LEED [43-45].

With disordered surfaces, the experimental challenge is to measure the diffuse intensity between any sharp spots due to the sub :irate. Such measurements have long been performed with Faraday cups. often as a by-product of measuring spot profiles. However, here again one welcomes automation to acquire larger amounts of experimental data. Video LEED has 
already been successfully applied to this task [6.33,38].

A more novel tool ideally suited to the measurement of diffuse LEED intensities is the position-sensitive detector $[46,47]$. This detector replaces the traditional LEED display screen with its requirement for conversion of an optical image into a digital set of data. Instead. one uses an electronic counting device which feeds its output directly in digital form into a computer: hence the name "digital LEED". To keep the counting rate down to a manageable value for the electronic counters, one uses nano-ampere to pico-ampere incident beam currents, and amplifies single electrons into detectable bursts through microchannel plates. The position-sensitive detector may be a resistive anode or a strip-and-wedge plate. In either case the detector outputs 3 or 4 analog curre.tts which are electronically combined to produce the position of the detected burst representing a single electron.

After recording of the experimental data by either video or digital LEED. they are manipulated to produce a suitable database for comparison with theory. In the case of ordered overlayers, a computer must identify the spots, make background corrections, integrate the spot intensities over their cross-sections and string together the data into I- $V$ curves.

The diffuse LEED intensities require some more treatment. Several new considerations arise, compared to spot intenaities $[6,33,34,38]$. First, because the diffuse intensities are typically weak. there is a danger that they are affected by inelastic intensity contributions. The enercy filtering that is normally applied in LEED does not excludo inelastic electrons which have suffered phonon losses or other energy losses of about $0.25 \mathrm{eV}$ or less. This issue has been studied by lbach and Lehwald [48]: they found that on the whole inelastic losses do not affect the measured diffuse intensities adversely. However, there are particular combinations of angles and energies where the elastic intensity is very low (due to destructive interference) while the inelastic losses are larger. One might apply a narrower energy-acceptance window. but this is not easy because of the smal! energy differences involved and the non-uniformities of the filtering grids. An other alternative, suggested by lbach and Lehwald, is to subtract the diffuse interisities of the clean surface from those of the overlayer-covered surface. At least those phonon losses common to both surfaces will then approximately cancel out.

Another concern with diffuse intensities goes back to our discussion in Sect. 3 about the structuie factor and the form factor. The diffuse intensities can be viewed as the product of an intensity due to the long-range order (the structure factor) and an intensity due to the short-range order (the form factor). If we are interested in the short-range order, i.e. the local bonding geometry, then we should eliminate the perturbing influence of the structure factor. because it is not normally included in the LFED theory: it could only be ireluded if one knew the long-range order in the first place. This is relatiyeiy easily accomplished in the case of purely two-dimensional disorder (thus excluding step disorder, for example). One can then use the logarithmic derivative of the diffuse intensity with respect to energy. keeping the parallel momentum transfer cor-stant [6.33,34,38]. Since the structure factor remains constant for censtant parallel mementum transfer. it disappears. But one must then also in the theory produce the logarithmic derivative of the calculated diffuse intensity: that is straightforward.

\section{Conclusions}

Our main conclusion is that the computational barrier to the structura! determination of complex and disordered structures has been largely broken down. The prohibitive power laws governing the computational cost as a function of complexity have been reduced to direct proportionality or even more favorable behaviors.

The only major remaining barrier to structural determination is the issue of how to search through the high-dimensional structural parameter space for the correct solution. This is the same issue facing $x$-ray crystallography. There are no universal mathematical methods that are effective at finding the correct structural solution in a unique and reliable manner in a high-dimensional parameter space. 
Thus, LEED crystallography has progressed to the the point where it is faced with the same problems that $x$-ray crystallography faces. However, $x$-ray crystallography benefits from the existence of "direct methods". which help in certain not-too-complex situations. These methods are based on the kinematic nature of $x$-ray diffraction and it is not yet clear whether similar methiods mighe be viable in the case of LEED.

LEED theory is now capable of treating not only surfaces with any large unit cell, but also disordered surfaces. In addition, incommensurate overlayers can now be treated effectively.

The next stage, already partly realized, is that of vacancies [49] and substitutional or interstitial impurities in surfaces. Also, steps at surfaces [24] and adsorbates attached to those steps are becoming accessible.

Other types of defects, such as local distortions. are already covered by present theoretical techniques.

On the experimental side, automated data acquisition systems are already available to handle the demand for larger data bases to solve more complex structures. Diffuse intensities due to disordered surfaces can also be measured, either with a video camera or. better. with a position-sensitive detector.

A word of caution is however needed with respect to disordered and defected surfaces. Their experimental preparation is more difficult than with ordered surfaces. because the sharp LEED pattern is no longer ayailable to judge the reproducibilty of then surface. In other words. it is more difficult to determine the state of a disordered or defected surface. It is therefore harder to reproduce desired surface conditions in such cases.

\section{Acknowledgements}

This work was supported in part by the Director, Office of Energy Research. Office of Basic Energy Sciences, Materials Sciences Division of the U.S. Department of Energy under Contract No. DE-AC03-76SF00098. Supercomputer time was also made available by the Office of Energy Ressarch of the U.S. Department of Energy. The NFEC development was in part funded by the Army Research Office. Many colleagues have contributed significantly to our work described in this text: J.J. Barton. G.S. Blackman. C.-M. Chan. 2.P. Hu. C.-T. Kao. R.J. Koestner. R.F. Lin. D.F. Ogletree. K. Ohtani. J.B. Fendry. P.J. Rous. D.K. Saldin. G.A. Somorjai. E. Sowa, and M.L. Xu 


\section{References}

1. J.B. Pendry, "Low-Energy Electron Diffraction". Academic Press (London) 1974.

2. M.A. Van Hove and S.Y. Tong, "Surface Crystallography by LEED", Springer-Verlag (Berlin. Heidelberg, New York) 1979.

3. M.A. Van Hove and G.A. Somorjai, "Adsorbed Monolayers on Solid Surfaces", Structure and Bonding, Vol. 38, Springer-Verlag (Berlin. Heidelberg, New York) 1979. 1985.

4. L.J. Clarke, "Surface Crystallography: An Introduction to LEED". J. Wiley (London)

5. M.A. Van Hove, W.H. Weinberg and C.-M. Chan, "LEED: Experiment, Theory and Structural Determination". Springer-Verlag (Berlin, Heidelberg. New York) 1986.

6. K. Heinz. D.K. Saldin and J.B. Pendry. Phys. Rev. Lett. 55.2312 (1985).

7. J.M. McLaren, J.B. Pendry, P.J. Rous, D.K. Saldin. G.A. Somorjai, M.A. Van Hove and D.D. Vvedensky, "Surface Crystallographic Information Service: A Handbouk of Surface Structures". D. Reidel (Dordrecht) 1987.

8. J.B. Pendry. "Surface Crystallographic Information Service: Database and Graphics Programs". D. Reidel (Dordrecht) 1987.

9. W. Moritz and D. Wolf, Surf. Sci. 163, L655 (1985).

10. C.-M. Chan and M.A. Van Hove, Surf. Sci. 171. 226 (1986).

11. E. Sowa. M.A. Van Hove and D.L. Adams, to be published.

12. C.-M. Chan and M.A. Van Hove. Surf. Sci. 183, 303 (1987).

13. E. Lang. K. Mülier. K.Heinz, M.A. Van Hove, R.J. Koestner and G.A. Somorjai, Surf. Sci. 127, 347 (1983).

14. W.S. Yang. F. Jona and P.M. Marcus, Phys. Rev. B28, 2049 (1983).

15. F.J. Himpsel. P.M. Marcus, R. Tromp, I.P. Batra, M.R. Cook. F. Jona and H. Liu. Fhys. Rev. B30, 2257 (1984).

16. S.Y. Tong. W.N. Mei and G. Xu. J. Vac. Sci. Technol. B2. 35.3 (1984).

17. R.F. Lin, G.S. Blackman. M.A. Van Hove and G.A. Somorjai. Acta Crys. B. in press.

18. H. Ohtani. M.A. Van Hove and G.A. Somorjai, to be published. (1970).

19. C.B. Duke and G.E. Laramoro. Phys. Rev. B2, 4765 (1970): Phys. Rey. B2, 4783 (1978)

20. W. Moritz. H. Jagodzinski and D. Wolf. Surf. Sci. 77. 233 (1978); Surf. Sci. 77, 249

21. J.B. Pendry, in "Determination of Surface Structure by LEED". P.M. Marcus and F. Jona. Eds.. Plenum (New York) 1984.

22. J.B. Pendry and D.K. Saldin, Surf. Sci. 145. 33 (1984).

23. D.K. Saldin. D.D. Vuedensky and J.B. Pendry, in "The Structure of Surfaces". M.A. Van Hove and S.Y. Tong, Eds., Springer-Verlag (Berlin. Heidelbe. ¿. New York) 1985. p.131.

24a. P.J. Rous and J.B. Pendry. Surf. Sci. 173.1 (1986): b. D.K. Saldin and J.B. Pendry. Surf. Sci. 162, 941 (1985).

25. N. Masud and J.B. Pendry. J. Phys. C9, 1833 (1976).

26. F. Jona, J.A. Strozier, Jr. and P.M. Marcus, in "The Structure of Surfaces". M.A. Van Hove and S.Y. Tong. Eds., Springer-Verlag (Berlin, Heidelberg, Wew York) 1985, p.92.

27a. M.A. Van Hove and G.A. Somorjai, Surf. Sci. 114, 171 (1982): b. M.A. Van Hove. R.J. Koestner. J.C. Frost and G.A. Somorjai, Surf. Sci. 129, 482 (1983).

28. M.A. Var Hove. R. F. Lin and G.A. Somorjai. J. Am. Chem. Soc. 108, 2532 (1986).

29. S.Y. Toi *. M.A. Van Hove and B.J. Mrstik, in Proc. 7th Int I Vacuum Congress and 3rd Int'I Conf. Solid Surfaces, Vienna. 2407 (1977)

30. J.J. Barton and M.A. Van Hove. Bull. Am. Phys. Soc. 31, 425 (1986): and to be published.

31. J.J. Barton and D.A. Shirley. Phys. rev. B32, 1906 (1985).

32. M.A. Van Hove. R.F. Lin and G.A. Somorjai. Phys. Rev. Lett. 51.778 (1983). 
33. P.J. Rous, J.B. Pendry, K. Heinz, K. Müller and N. Bickel. Phys. Rev. Lett. 57, 2951 (1985).

35. J.J. Barton. M.L. Xu and M.A. Van Hove, to be published.

36. M.L. Xu. J.J. Barton and M.A. Van Hove, to be published.

37. W.F. Egelhoff. Jr., Phys. Rev. B30. 1052 (1984): W.F. Egelhoff, Jr.. J. Vac. Sci. Technol. A3. 1511 (1985): R.A. Armstrong and W.F. Egelhoff, Jr., Surf. Sci. 154. L225 (1985).

38. K. Heinz, K. Müller, W. Popp and H. Lindner, Surf. Sci. 173, 366 (1986). (1987).

39. Z. P. Hu. D.F. Oglatree, M.A. Van Hove and G.A. Somorjai. Surf. Sci. 180, 433

40. D.F. Ogletree, M.A. Van Hove and G.A. Somorjai. Surf. Sci. 183.1 (1987).

41. C.-T. Kao, G.S. Blackman, C.M. Mate, B.E. Bent. M.A. Van Hove and G.A. Somorjai. to be published.

42. G.S. Blackman. C.M. Mate, M.A. Van Hove and G.A. Somorjai. to be published.

43. P. Heilmann. E. Lang, K.Heinz and K. Müller. Appl. Phys. 19. 247 (1976).

44. E. Lang, P. Heilmann. G. Hanke. K. Heinz and K. Müller. Appl. Phys. 19. 287 (1979).

45. D.F. Ogletree, G.A. Somorjai and J.E. Katz. Rev. Sci. Instr. 57. 3012 (1986).

46. P.C. Stair, Rev. Sci. Instrum. 51, 132 (1980).

47. D.F. Ogietree, G.A. Somorjai and J.E. Katz, to be published.

48. H. Ibach and S. Lehwald. Surf. Sci. 176, 629 (1986).

49. P.J. Rous and J.B. Pendry. Surf. Sci. 155. 241 (1985). 
Table 1. Dependence of the cost of conventional LEED computations on basic parameters

$\begin{array}{lc}\text { in } & \text { in } \\ \text { spherical- } & \text { plane-wave } \\ \text { wave space } & \text { space }\end{array}$

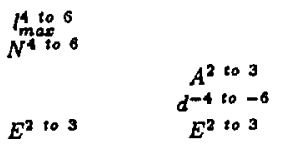

$$
\begin{aligned}
& I_{\text {max }}=\text { cutoff angular momentum } \\
& N=\text { no, atoms in unit cell } \\
& A=\text { unit cell area ( } \propto \text { no. beams) } \\
& d=\text { interlayer spacing (small for steps) } \\
& E=\text { kinetic energy }
\end{aligned}
$$


Figure Captions

Fig. 1. Structural diagram of reconstructed fcc $\{110\}-(1 \times 2)$, representative of the corresponding Au, Ir and Pt surface structures

Fig. 2. Perspective view of sulfur adatoms adsorbed on reconstructed $\operatorname{Ir}\{110\}-(1 \times 2)$.

Fig. 3. Side yiew (top panel) and top view (main panel) of $\mathrm{Rh}\{111\}-(3 \times 3)-\mathrm{C}_{6} \mathrm{H}_{6}+2 \mathrm{CO}$. Van der Waals contours are given for the molecules. The hydrogen positions are assumed. $A(3 \times 3)$ unit is outlined. Selected distances are shown, indicating a large $C_{6}$ ring distortion relativo to the gas-phase $\mathrm{C}_{6} \mathrm{H}_{8}$ molecule (for which the $\mathrm{C}-\mathrm{C}$ distances are all $1.397 \AA$ )

Fig. 4. Similar to Figure 3, but for Pd as a substrate rather than Rh. Note the minor $C_{B}$ ring distortion in this case relative to the Rh case. Also, the molecules in this case have adopted different hollow sites than on $\mathbf{R h}$

Fig. 5. Schematic representation of two clusters built around two different surface atoms. The two spherical clusters overlap in the sense that some scattering paths are common to both clusters

Fig. 6. The distinction between lattice and basis is emphasized, together with the different implications for electron diffraction

Fig. 7. The principle of near-neighbor multiple scattering is exhibited by an example of an included path (left diagram) and an example of an excluded path (right diagram). The scattering cluster is taken as a hexagonal ring of atoms. An electron is allowed to scatter only between nearest neighbors in the NNMS method

Fig. 8. The two beam sets used in the beam-set-neglect method are shown. In the top diagram, the dots represent beams (plane waves) obtained from the incident beam by diffraction from the substrate lattice. The crosses represent a similar set of beams obtained from the outgoing detected beam. These two sets are schematically shown in the bottom diagram, emphasizing scattering at the overlayer and separately at the substrate

Fig. 9. Calculated I-V curves for a layer of benzene on $\mathrm{Rh}\{111\}$ with a (3×3) periodic superlattice. The curves marked "clean" coirespond to the adsorbate-free substrate. The curves marked " $(3 \times 3)^{*}$ are due to an all-beam calculation: they can be viewed as "exact" (except for the constant neglect of multiple scattering in the overlayer in all cases shown here). The other curves are due to various levels of beam set neglect. as described in the text

Fig. 10. I-V curves calculated with and without beam set neglect are compared with experiment for benzene on $R h\{111\}$, by means of an $R$-factor. The interlayer spacing between substrate and molecules is varied to show the effect of BSN on structural determination. Two adsorption sites (CABC hollow and $b A B C$ hollow) are tested

Fig. 11. Diffuse LEED intensities calculated for disordered oxygen atoms in hollow sites on $\mathrm{Ni}\{100\}$. for two different $\mathrm{Ni}-\mathrm{O}$ interlayer spacings. The spikes represent sharp integral-order substrate-induced beams (not to scale in either width or height). The grazing-emergence condition gives rise to the intensity cut-off forming a circle around the specular reflection direction. Normal incidence is assumed. giving four-fold rc:ational symmetry and mirror planes 
Fig. 12. Structural diagram of a graphite layer adsorbed on $P_{t}\{111\}$, with an interralated chemisorbed carbon layer. The graphite layer is incommensurate with the substrate, while the individual carbon atoms are bonded in hollow sites of the substrate

Fig. 13. Diffuse diffraction by a disordered overlayer of molecules. The large circles represent the clusters within which multiple scattering is important for the molecule at its center. When the molecules are tightly packed, giving overlapping spheres, electrons can. scatter from one molecule to the other, damaging the kinematic-sublayer-addition method 
fcc $(110)-(1 \times 2)$ missing-row model

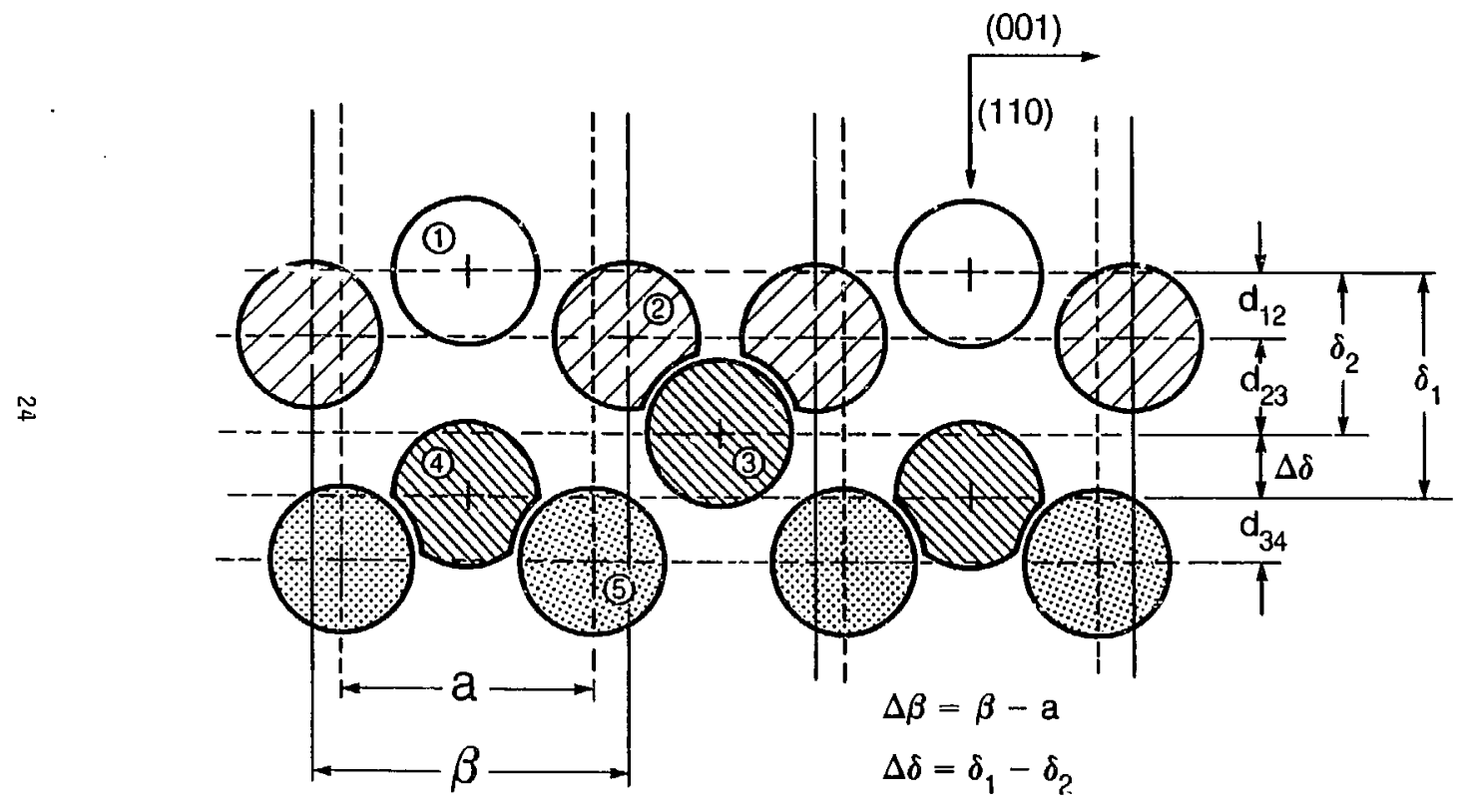




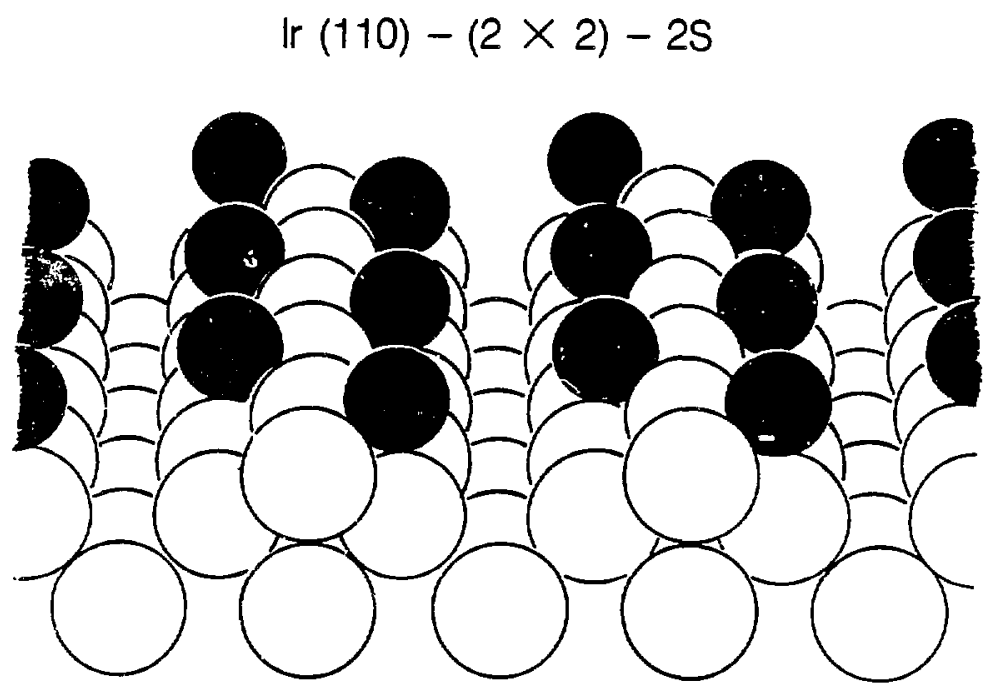

XEL 867-10137 


\section{Near-Neighbor Multiple Scattering (NNMS)}
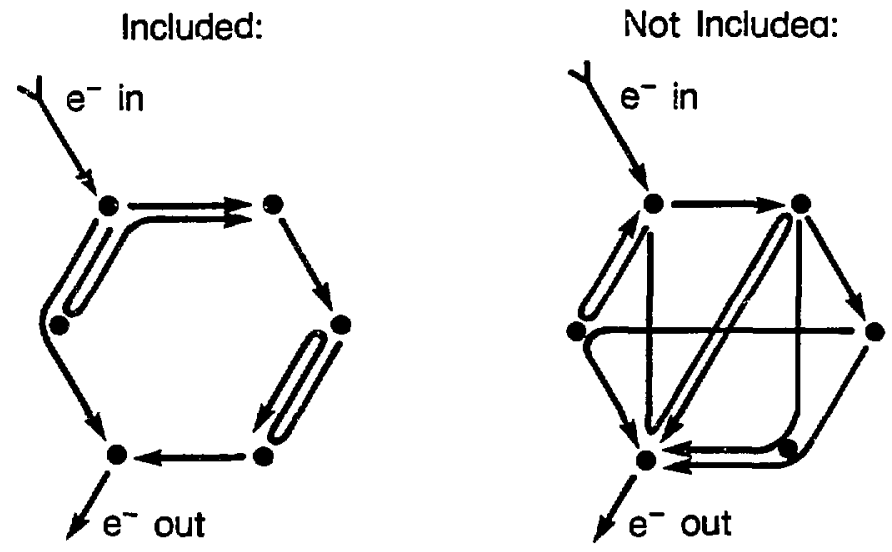

XBL 877.9741 


\section{Diffraction: Lattice vs. Basis}

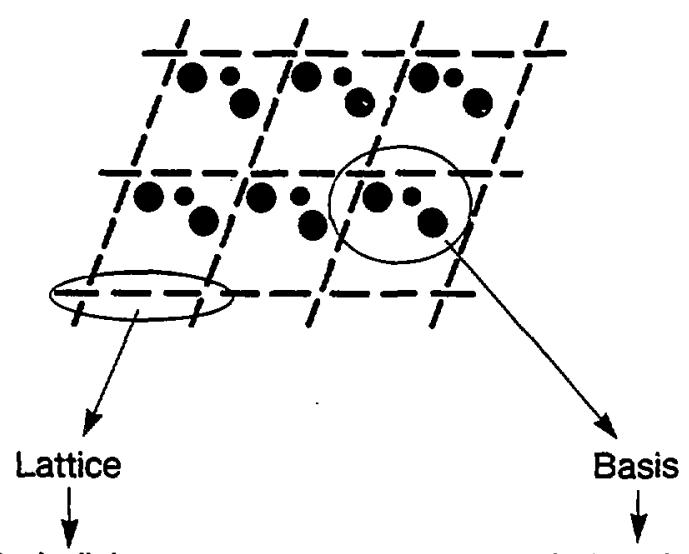

Periodicity

Unit Cell Shape, Size, Orientation

Long-Range Order

LEED Spot Pattern

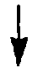

"Kinematic"
Relative Atomic Positions Unit Cell Contents Short-Range Order

LEED Spot Intensities

"Dynamical" 


\section{Kinematic Cluster Addition}

For Overlapping Clusters

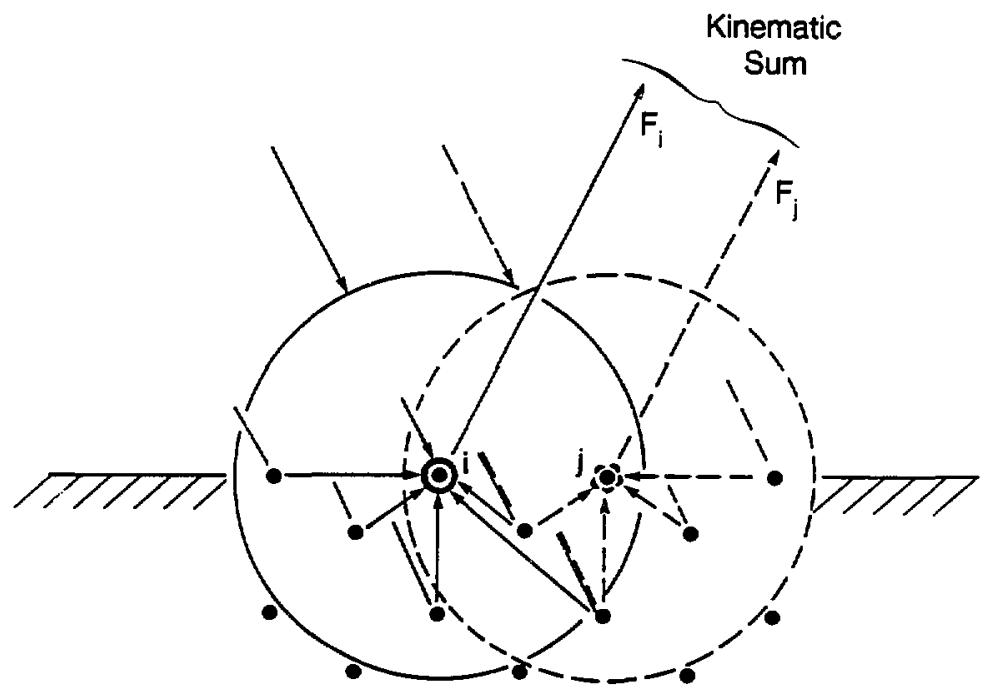



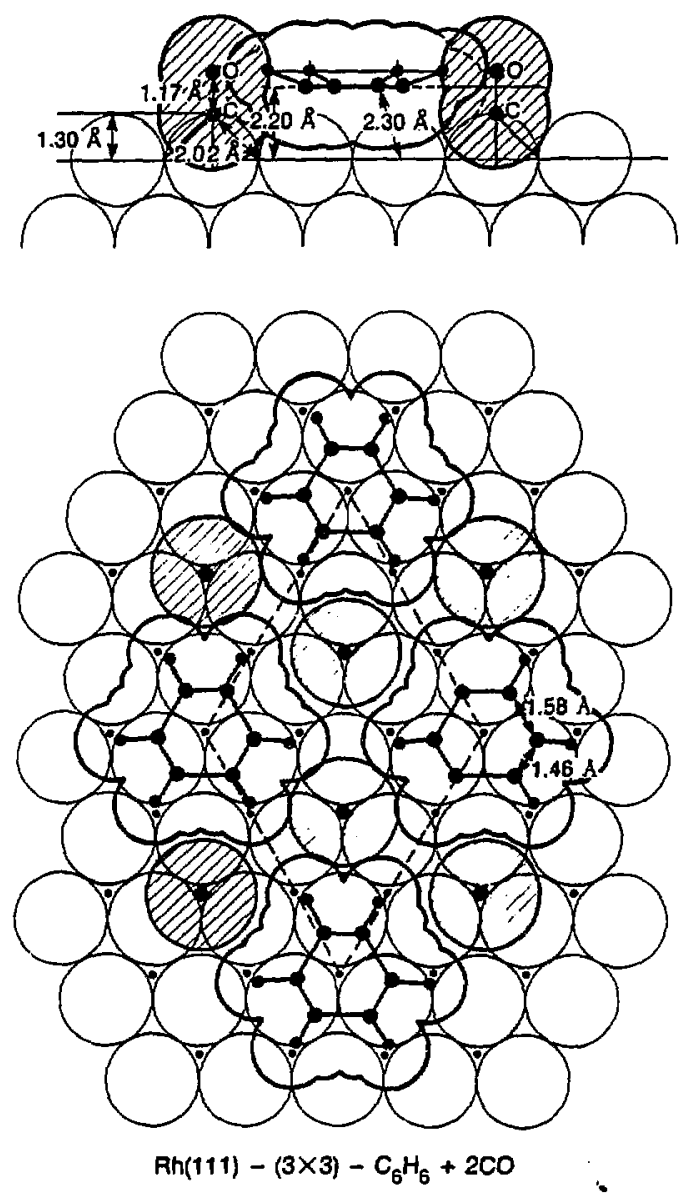

XEL 860.10701 
Beam Set Neglect
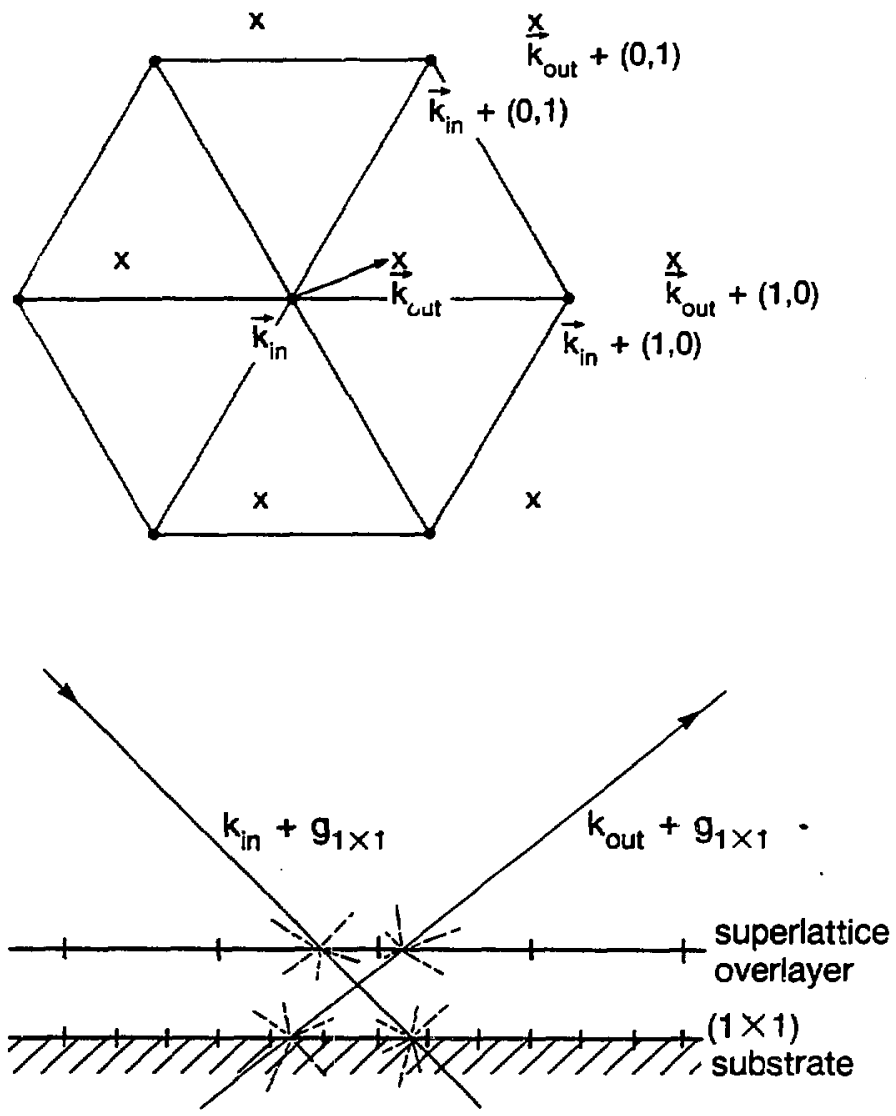

X日L 877.7001 


\section{Effect of BSN on R Factor Values}

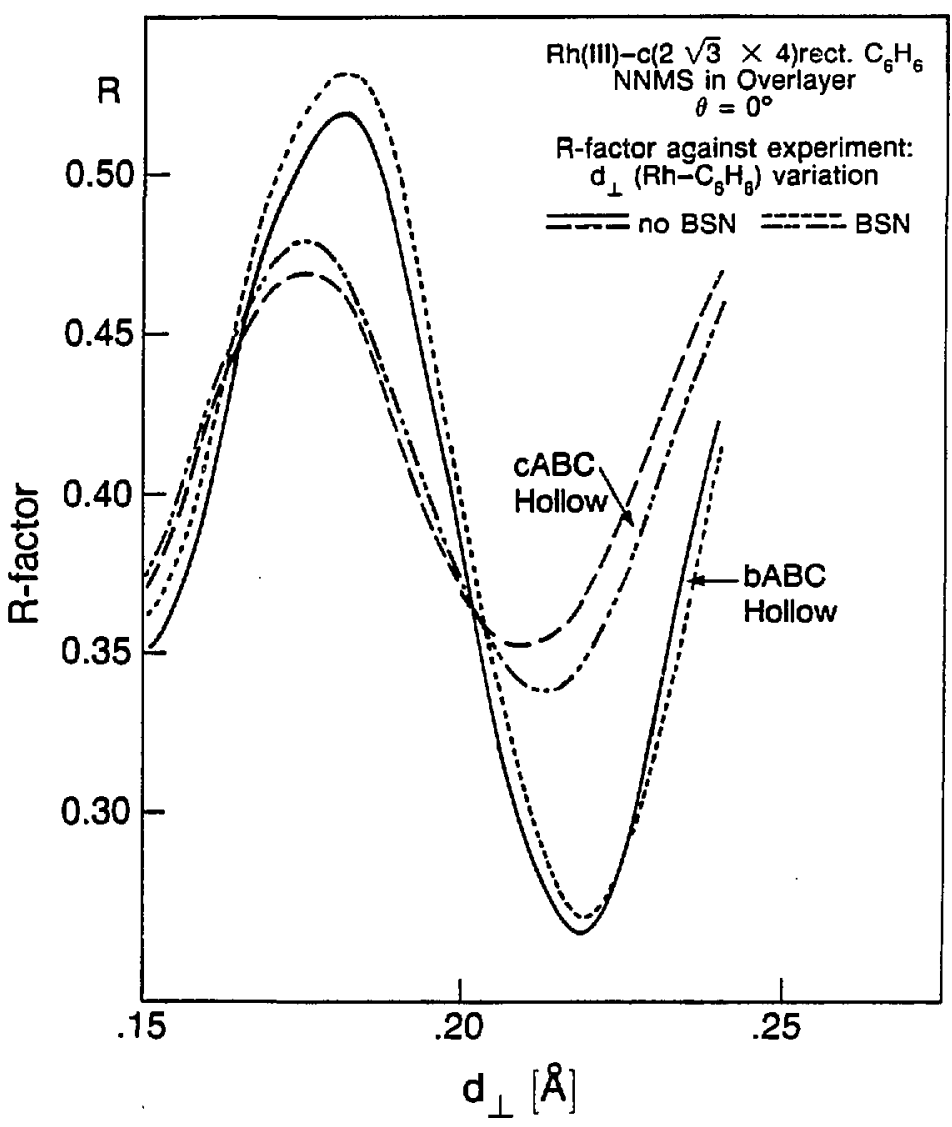

XBL 877.9742 


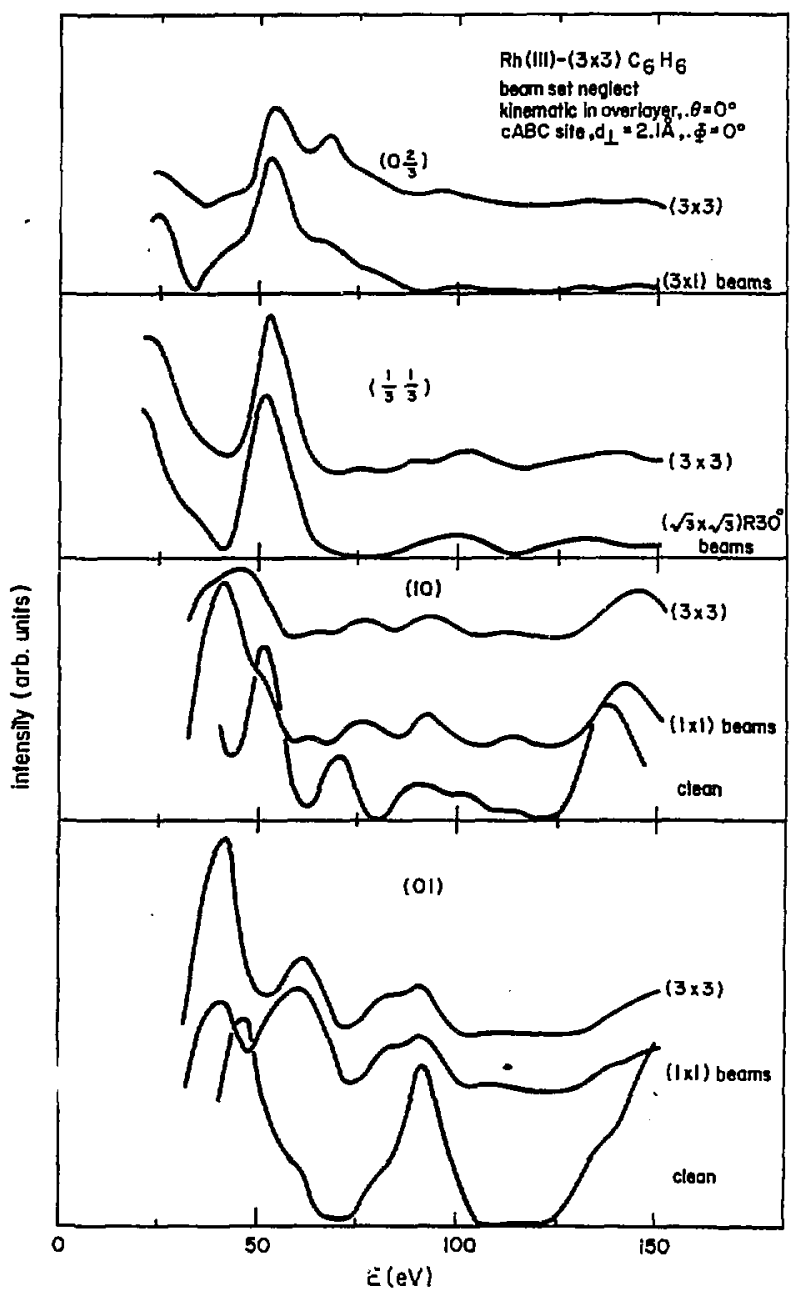

XBL $035-199$ 


\section{Diffuse Elastic LEED}

For $\mathrm{O} / \mathrm{Ni}(100)$ as Function of $d_{\perp}(\mathrm{Ni}-\mathrm{O})$
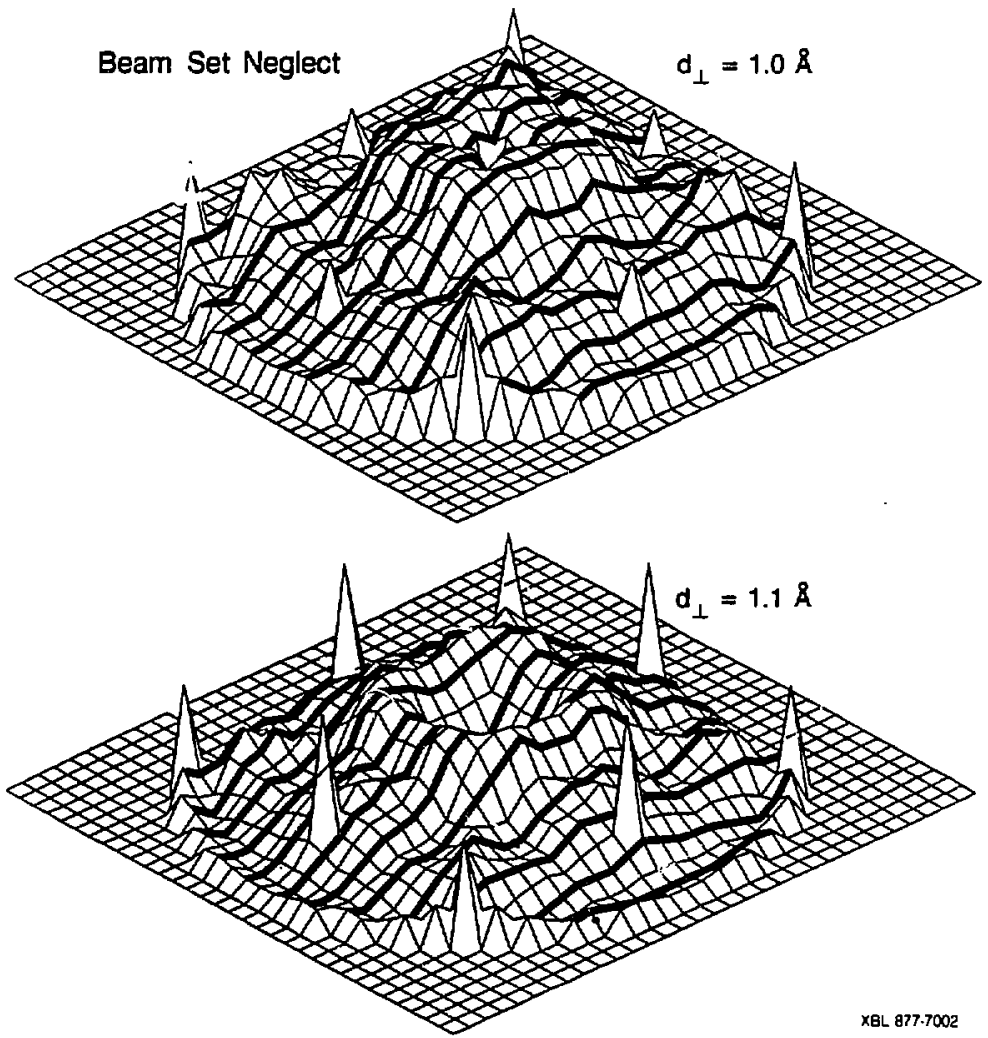


\section{Incommensurate graphite \\ on $\mathrm{Pt}(111)$ \\ with $\mathrm{C}$ intercalate}

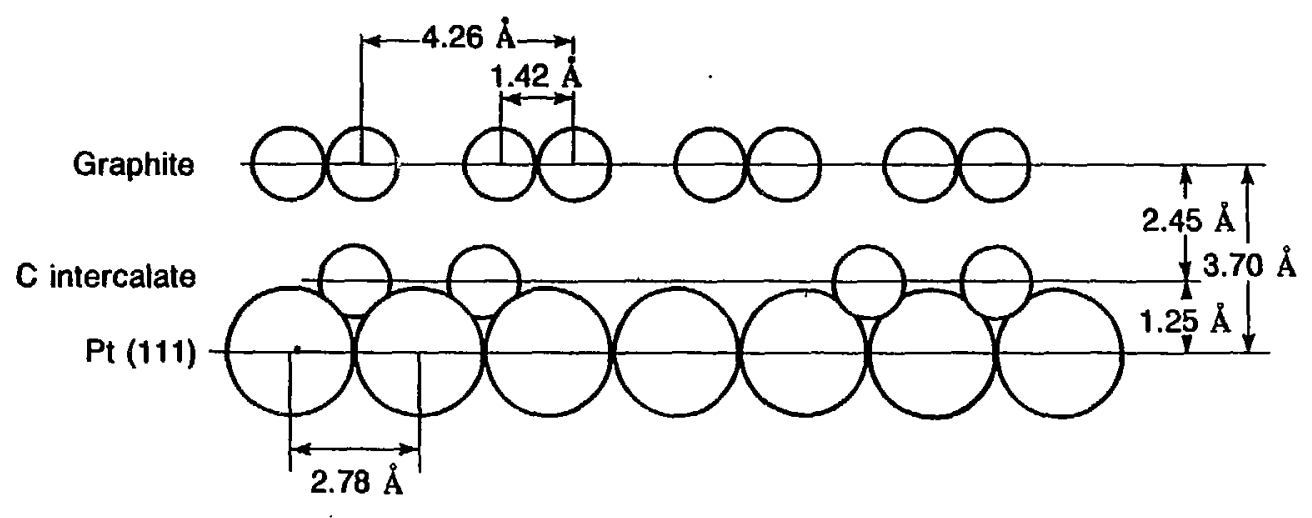

X8L 8512-12785 


\section{Diffraction By Disordered Array}

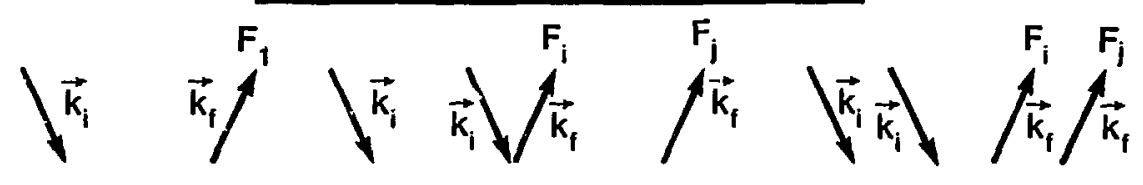

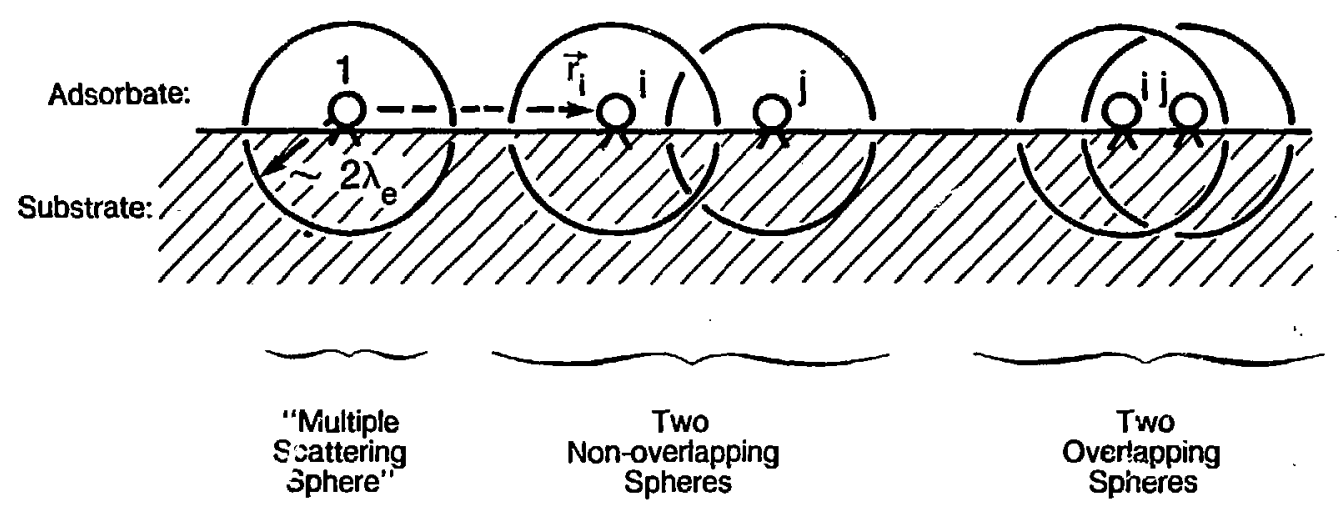




\section{DISCLAIMLR}

This report was prepared as an account of work sponsored by an agency of the United States Government. Neither the United States Government nor any agency thereof, nor any of their employocs, makes any warranty, express or implied, or assumes any legal liability or responsibility for the accuracy, completeness, or usefulness of any information, apperatus, product, or process disclosed, or represents that its use would not infringe privately owned rights. Reference herein to any specific commercial product, proces, or service by trade name, trademark, manufacturer, or otherwise does not nocessarily constitute or imply its endorsement, recommendation, or favoring by the United States Goverament or any agency thereof, The views and opinions of autbors expressed herein do not necessarily state of reflect those of the United States Government or any agency thereof. 